\title{
Molecular network topology and reliability for multipurpose diagnosis
}

This article was published in the following Dove Press journal:

International Journal of Nanomedicine

18 October 2011

Number of times this article has been viewed

MA Jalil'

N Moongfangklang ${ }^{2,3}$

K Innate ${ }^{4}$

S Mitatha ${ }^{3}$

J Ali ${ }^{5}$

PP Yupapin ${ }^{4}$

'Ibnu Sina Institute of Fundamental Science Studies, Nanotechnology Research Alliance, University of Technology Malaysia, Johor Bahru, Malaysia; ${ }^{2}$ School of Information and Communication Technology, Phayao University, Phayao, Thailand; ${ }^{3} \mathrm{Hybrid}$ Computing Research Laboratory, Faculty of Engineering, King Mongkut's Institute of Technology Ladkrabang, Bangkok, Thailand; ${ }^{4}$ Nanoscale Science and Engineering Research Alliance, Advanced Research Center for Photonics, Faculty of Science, King Mongkut's Institute of Technology Ladkrabang, Bangkok, Thailand; Institute of Advanced Photonics Science, Nanotechnology Research Alliance, University of Technology Malaysia, Johor Bahru, Malaysia
Correspondence: PP Yupapin

Nanoscale Science and Engineering Research Alliance, Advanced Research Center for Photonics, Faculty of Science, King Mongkut's Institute of Technology Ladkrabang, Bangkok 10520, Thailand

Tel +66 23298414

Fax +66 23298410

Email kypreech@kmitl.ac.th
Abstract: This investigation proposes the use of molecular network topology for drug delivery and diagnosis network design. Three modules of molecular network topologies, such as bus, star, and ring networks, are designed and manipulated based on a micro- and nanoring resonator system. The transportation of the trapping molecules by light in the network is described and the theoretical background is reviewed. The quality of the network is analyzed and calculated in terms of signal transmission (ie, signal to noise ratio and crosstalk effects). Results obtained show that a bus network has advantages over star and ring networks, where the use of mesh networks is possible. In application, a thin film network can be fabricated in the form of a waveguide and embedded in artificial bone, which can be connected to the required drug targets. The particular drug/nutrient can be transported to the required targets via the particular network used.

Keywords: molecular network, network reliability, network topology, drug network, multiaccess network

\section{Introduction}

Network topology is recognized as an important tool for network design and applications, especially in large area networks where the network stability and reliability are vital for network performance. The use of such tools is also applicable for more complex systems, especially when the size of network devices and components are reduced to nanoscale regimes. Nanoscale devices and networks can be used as molecular networks for applications in such areas as biomedical engineering, ${ }^{1}$ health care, ${ }^{2}$ pharmaceuticals, ${ }^{3}$ and nanomedicine. ${ }^{4,5}$ The use of a medical information bus (MIB) standard ${ }^{6,7}$ for the bidirectional interconnection of medical devices and computers was first proposed in 1984 and approved by the Institute of Electrical and Electronics Engineers and the American National Standards Institute in 2000-2001 for digital communication networks. Extensive research has improved and supported the MIB standard. ${ }^{8-10}$ Recently, the use of molecular buffer and bus networks for the diagnosis of Alzheimer's disease has been proposed ${ }^{4}$ and a multi-access drug delivery network and stability of this has been analyzed. ${ }^{5}$ The required molecular volumes for drug or protein can be trapped and moved dynamically within the molecular bus network. The advantage of the system is that the diagnostic method can be performed within the small system, which is available as a human-embedded device for diagnostic use.

Reliability plays an important role in realistic applications and in transporting large microscopic volumes through the network. One study has investigated the 
performance and efficiency of network topologies. ${ }^{11}$ The efficiency power budget of optical bus and star networks have been analyzed by determining the number of stations that can be supported with and without erbium-doped fiber optical amplifiers in single folded bus, double bus, and star networks. For large-scale networks, the performance of optical network topologies of optimized semiconductor optical amplifiers was analyzed and compared. ${ }^{12}$ Bus, ring, star, and tree network topologies were investigated for differential phase shift keying $10 \mathrm{Gbit} / \mathrm{s}$, where the quality factor and power saturation are considered. The performance of a network system is evaluated in terms of the number of users supported at the minimum signal input power of $-40 \mathrm{dBm}$. The comparison of optical network topologies for wavelength division multiplexing transport networks ${ }^{13}$ was investigated for the various network topologies. Pountourakis et a $1^{14}$ evaluated the performance by receiver collisions analysis in high-speed optical fiber local-area networks using star topology. The quantitative comparison of the error-containment capabilities of bus and star topology in a controller area network ${ }^{15}$ was also investigated. From the above details, there is little information available on molecular network reliability. Similarly, when the signal is flown within the micro-/nanoscale network, it is important to ascertain the network reliability. Therefore, the study of molecular network reliability is necessary for large microscopic volumes and networks to avoid gene/molecule transportation problems in the network.

In this paper, the use of network topology for molecular network design is proposed. Signal transmission, in terms of signal to noise ratio (SNR) and crosstalk effects, is calculated to assess network reliability.

\section{Molecular network topologies}

The basic trapping tool known as an optical tweezer is used to trap and transport the molecule within the network. Theoretically, the trapping force is induced by the gradient field of the light pulse. The trapping forces are exerted by the intensity gradients in the strongly focused light beam to trap and move the microscopic volumes. The optical forces are customarily defined by the relationship: ${ }^{16}$

$$
F=\frac{Q n_{m} P}{c}
$$

Here, $Q$ is a dimensionless efficiency, representing the fraction of power utilized to exert force, $n_{m}$ is the refractive index of the suspending medium, $c$ is the speed of light, and $P$ is the incident laser power, measured at the specimen.
For a plane wave incident on a perfectly absorbing particle, $Q$ is equal to 1 . To achieve stable trapping, the radiation pressure must create a stable, three-dimensional equilibrium. As biological specimens are usually contained in aqueous medium, the dependence of $F$ on $n_{m}$ can rarely be used to achieve strong trapping forces. The increase in laser power is possible but only over a limited range due to the possibility of optical damage. $Q$ itself is therefore the major parameter of trapping force. In the Rayleigh regime, ${ }^{4,5}$ the trapping forces decompose naturally into two components as the scattering force and gradient force. Since, in this limit, the electromagnetic field is uniform across the dielectric, the particles can be treated as induced point dipoles. The scattering $\left(F_{\text {scatt }}\right)$ and the gradient field $\left(F_{\text {grad }}\right)$ are the Lorentz force that acts on the dipole, induced by the light field as defined by Jalil ${ }^{16}$ and Neuman, ${ }^{17}$ which can be formed within the small system, for instance a nanoring resonator.

The input trapping force for each topology is generated and controlled in the form of a Gaussian pulse within the PANDA ring resonator by the control port signals. The $E_{t 1}$ and $E_{t 2}$ represent the optical fields of the through port and drop ports, respectively, as shown in Figure 3. All parameters are defined and described by Svoboda et al. ${ }^{18}$ The electric fields $E_{0 R}$ and $E_{0 L}$ are the field circulated within the nanoring at the right and left side of the add-drop optical filter. Moreover, in the design of this system a molecular buffer is required in the system, which can be used to store or delay atoms/ molecules for a period of time sufficient for operation. The light intensity and velocity can also be controlled ${ }^{19}$ and used for medical applications. Several researchers have shown that the use of fluid particles (drug volumes) can result in realistic applications. ${ }^{20}$

In this work, the use of an optical network principle to estimate network stability and reliability is proposed. In general, the major problems of large networks are reduced stability, insertion loss, and crosstalk effect (fiber channel). The insertion loss reduces the efficient transmission distance. Normally, in a popular communication network in which the signals pass through the "router," it is $5 \mathrm{~dB}$. According to the performance of the proposed point-to-point transmission system, a required network can be built over at least $50 \mathrm{~km}$. In future, along with the development of dense wavelength division multiplexing technology, it will be possible to reduce the insertion loss to less than $1 \mathrm{~dB} .{ }^{21}$ The multivariable network will be able to cover more than $100 \mathrm{~km}$ with high capacity. The crosstalk effect is mainly due to a signal of co-channel interference and adjacent-channel interference, resulting in bit errors in the system. The crosstalk can be 
considered in terms of channel separability. For a network, crosstalk brings bit errors, which must be reduced to a very low level. Insensity loss and fiber coupling loss can be estimated as:

$$
\begin{gathered}
I L=10 \times \log \left(\frac{P_{\text {in }}}{P_{\text {out }}}\right) \\
F C_{j}\left(\lambda_{i}\right)=10 \times \log \left[\frac{P_{j}\left(\lambda_{i}\right)}{P_{i}\left(\lambda_{i}\right)}\right]
\end{gathered}
$$

Here, $P_{\text {in }}$ and $P_{\text {out }}$ are input and output intensities; $P_{j}\left(\lambda_{i}\right)$ is output intensity with wavelength $\lambda$, which exports from port $j ; P_{i}\left(\lambda_{i}\right)$ is output intensity with wavelength $\lambda_{i}$, which exports from port i. $P_{i}\left(\lambda_{i}\right)$ in Equation 3 is equal to $P_{\text {out }}$ in Equation 2.

$$
\begin{gathered}
\frac{P_{i}\left(\lambda_{i}\right)}{P_{\text {in }}}=\frac{P_{i}\left(\lambda_{i}\right)}{P_{\text {out }}} \times \frac{P_{\text {out }}}{P_{\text {in }}}=10 \frac{\left[F C_{j}(\lambda i)-L L\right]}{10} \\
P_{\text {out }} / P_{\text {in }}=10^{-I L / 10}
\end{gathered}
$$

It is assumed that all input photons from any user are the same when they enter the router. Since a light pulse can pass through two add-drop filters when it passes a router, then the FC versus efficient signals is given as:

$$
\left[\frac{P_{i}\left(\lambda_{i}\right)}{P_{i n}}\right]^{2} \times\left[\frac{P_{\text {in }}}{P_{\text {out }}}\right]^{2}=10^{\frac{2 \times F C_{j}(\lambda i)}{10}}
$$

Now consider the situation where it is assumed that the input light pulse is not equal. Here the input photons have $\mathrm{X}$ $\mathrm{dB}$ insertion loss before they pass through a router. But light pulses that produce crosstalk do not produce any insertion loss. Thus, the ratio in Equation 6 will become $10^{\left[X+2 \times F C_{j}\left(\lambda_{i}\right)\right] / 10}$. If there are many inputs that produce crosstalk, the ratio must be

$$
\sum_{j=1}^{N-1} 10 \frac{\left[X+2 \times F C j\left(\lambda_{i}\right)\right]}{10}
$$

Usually, the SNR has the general applicability to the analysis of sensory discrimination (by nerve cells and by whole organisms) and to the performance of networks. Therefore, in this paper, SNR analysis is used to characterize or compare the level of desired signal with the level of background noise for reliability of neural information transmission. It is defined as the ratio of signal power (PS) to the noise power $(\mathrm{PN})$ in decibel values as shown in Equation 8.

$$
S N R=10 \times \log _{10}\left(\frac{P_{S}}{P_{N}}\right)
$$

A star network applied to a delivery system ${ }^{4,22}$ is most commonly used in computer networks. This network features a central connection point called a hub. In this study, it is replaced by a molecular buffer or router and each network node is replaced by neural cells as shown in Figure 1, which consist of four nanodevices (add/drop filters) before reaching the neuronal cells. First, a device called a "PANDA ring resonator" is used to generate the potential well for trapping the drug molecule. Second, the drug molecules are stored in the storage unit. Third, a molecular buffer functions as a molecular router to distribute the drug molecule to the targets (neuronal cells). Finally, a suitable molecule size can be tuned and obtained by the add-drop filter and required targets. The amplification part shows the example of kinesin and dynein molecule transportation, where the waveguide is connected to the microtubule of neuronal cells, as reported by Mitatha et al. ${ }^{4}$ The microgel/nanogel was used to connect the nanodevice and neuronal cells.

Devices typically connect to the hub, which acts as a conduit to transmit messages. If the central connection point is passive, the originating node must be able to tolerate the reception of an echo of its own transmission, delayed by the two-way transmission time plus any delay generated in the central connection point (hub). The active star network has an active central connection point to solve echo-related problems. The advantage of this topology is that a failure in any node in a star network cable will only take down those failing nodes, not the entire network. However, the optical star topology distributes the optical power equally to the output port and the number of users supported by the star is less than 64 nodes. ${ }^{23}$ Figure 2 shows a ring network in which every node has exactly two adjacent neighbors for communication purposes. All messages travel through a ring in the one direction (clockwise/counterclockwise). The disadvantage of this topology is that a failure in any cable or node breaks the loop and takes down the entire network. In employing a ring network, a fiber distribution data interface, synchronous optical network, or token ring technology is typically used to avoid problems. Normally, due to the power budget problem in the link, a limited number of nodes in a typical ring (less than six users at a bit rate 2.5 Gbits $/ \mathrm{s}^{24}$ ) is used. Ross ${ }^{25}$ has reported a ring network that can accommodate 25 nodes by using erbium-dope fiber amplifiers with a narrowband channel dropping at the same bit rate. The last proposed network is a bus network, as shown 


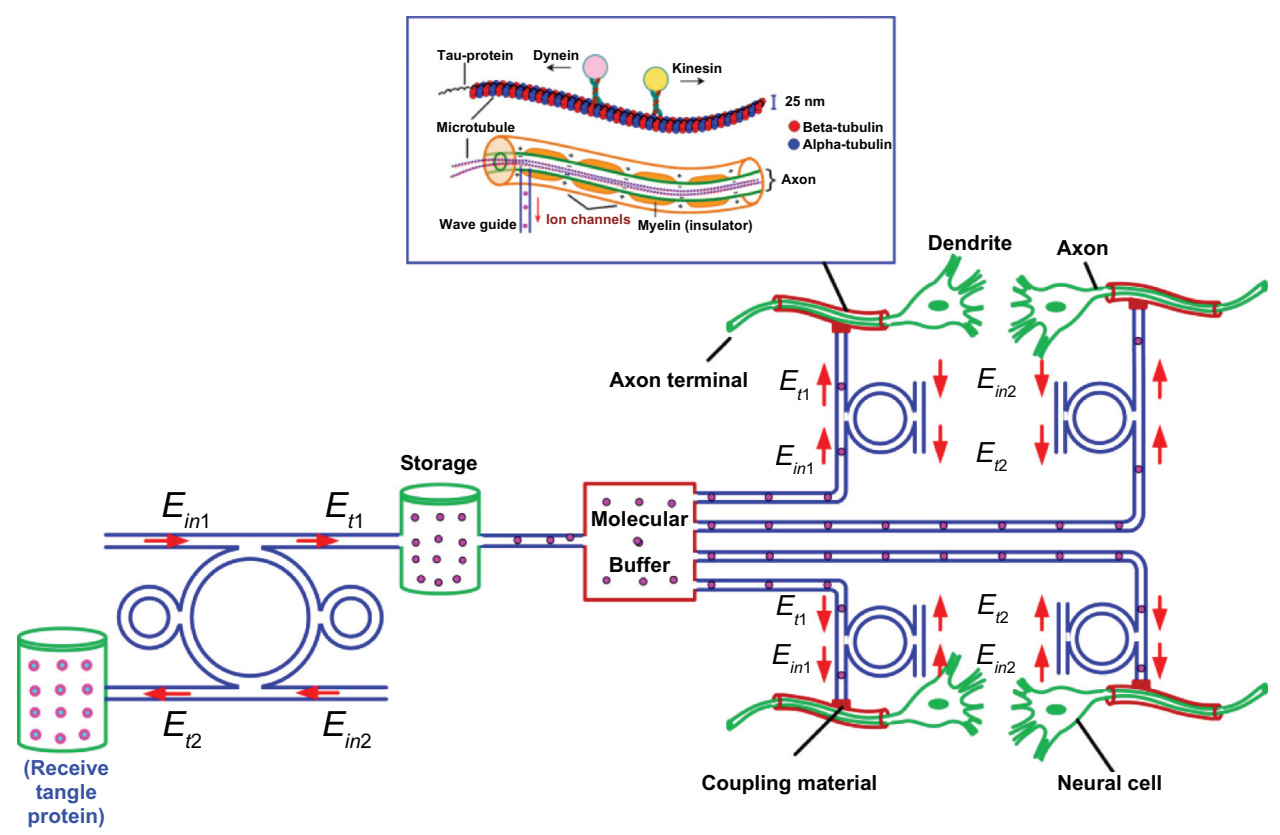

Figure I Schematic diagram of molecular star network, comprising four nanodevices before reaching the neuronal cells.

in Figure 3, in which a common backbone or trunk is used to connect all nodes in a network in a linear shape. A single cable functions as the shared communication medium for all the nodes attached with this cable with an interface connector. The node communicates to all outer nodes attached with the shared cable but only the intended recipient actually accepts and processes the message. The advantages of a bus network are that it is easy to install and requires fewer cables, because only a main shared cable is used for the network. However, bus networks work best with a limited number of node users and less than 20 nodes, as described by Willner and Hwang. ${ }^{26}$ If more than a few dozen nodes are added to a bus network, performance becomes a problem.

In this case, it is assumed that the spherical particle is polystyrene ( $\mathrm{n}=1.5894)$, the liquid medium is water ( $\mathrm{n}=1.33$ ), and the optical power, which is required to trap particles of a certain size/polarizability, is $9.1 \mathrm{~W}$. In simulation, an add/drop filter is a linear device, which means that the difference in phase can be neglected. The Gaussian pulse with center wavelength at $500 \mathrm{~nm}$, peak power $2 \mathrm{~W}$,

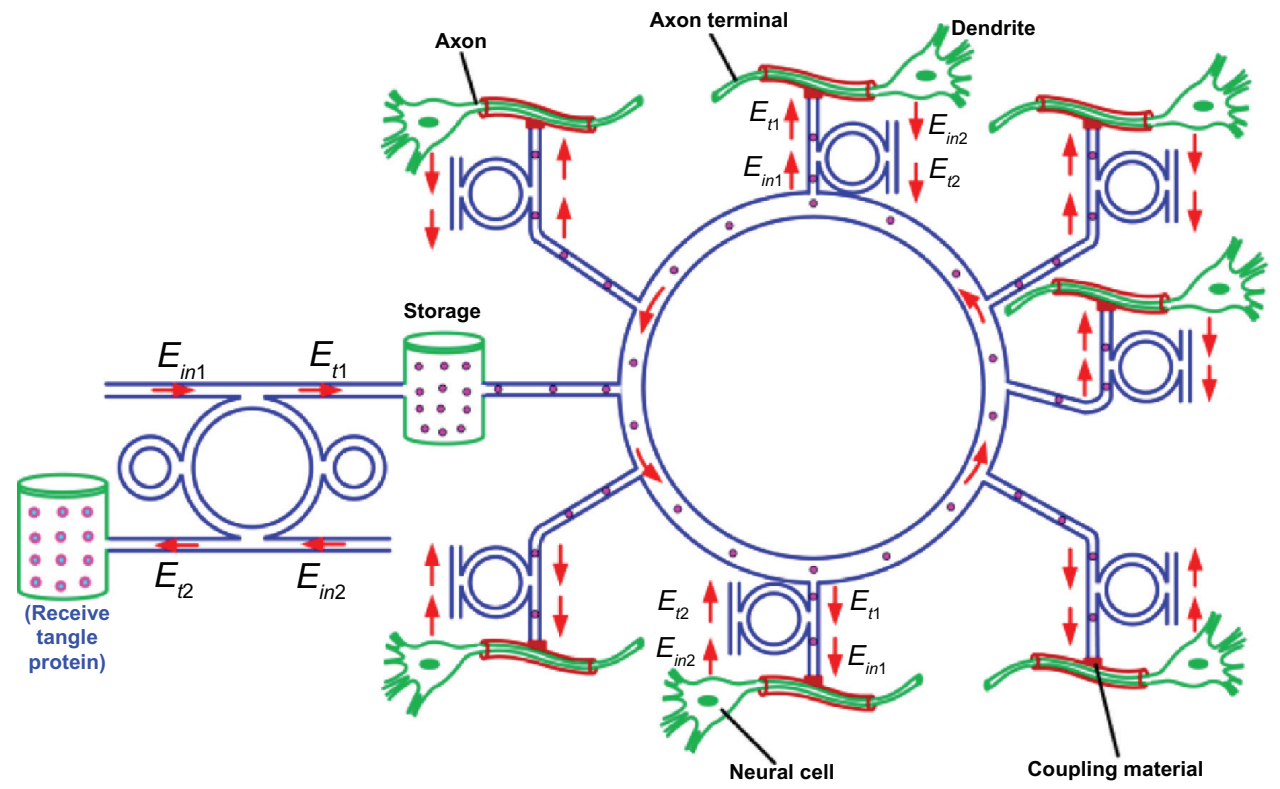

Figure 2 Schematic diagram of molecular ring network. 


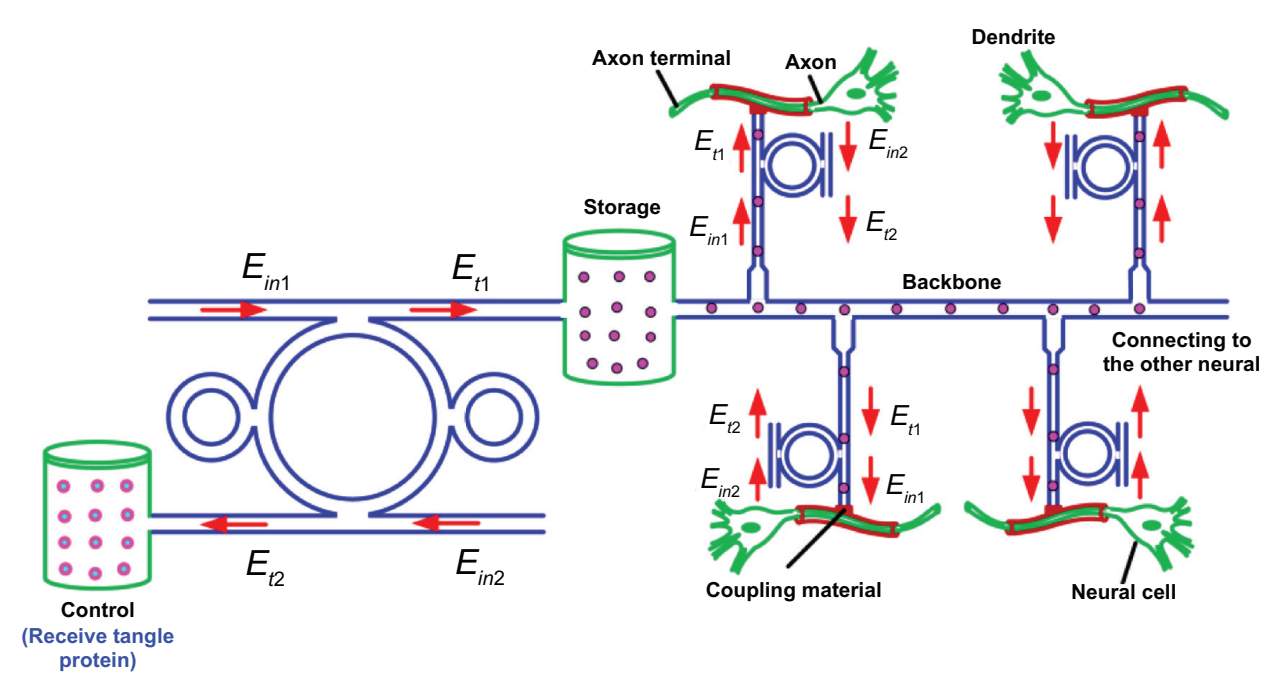

Figure 3 Schematic diagram of molecular bus network.

and pulse width of $35 \mathrm{fs}$ is inputted into the system via the input port. The control signal is bright soliton, where the coupling coefficients are given as $\kappa_{0}=0.5, \kappa_{1}=0.35, \kappa_{2}=0.1$, and $\kappa_{3}=0.35$, respectively. The ring radii are $R_{\text {add }}=20 \mu \mathrm{m}$ and $R_{R}=R_{L}=5 \mu \mathrm{m}$, respectively. Evidence on the practical device with the radius of 2 to $3 \mu \mathrm{m}$ has been reported by Piyatamrong et a ${ }^{27}$ and $A_{\text {eff }}$ is $200 \mu \mathrm{m}^{2}$. The dynamic tweezers (gradient fields) in the form of Gaussian pulse can be used to trap the required microscopic volume. Four different
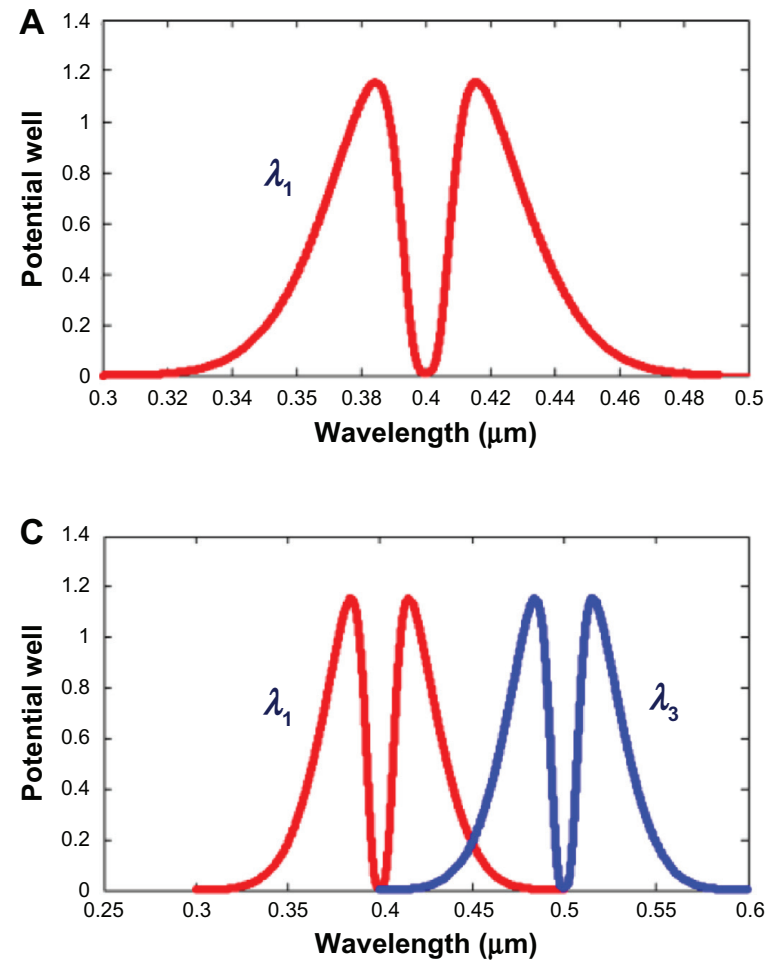

center wavelengths of tweezers are generated, in which the dynamic movements of trapped particles appear, as shown in Figure 4. The molecular trapping probe can be adjusted to fit the drug molecule size from 10 to $15 \mathrm{~nm}$, for drug molecule transportation at the through port and networks as shown in Figure 5. The number of molecules can be increased within the PANDA ring resonator (as shown in Figure 5A). In addition, the trapping tool (probe) or dynamic well size can be adjusted by varying the coupling coefficient of the
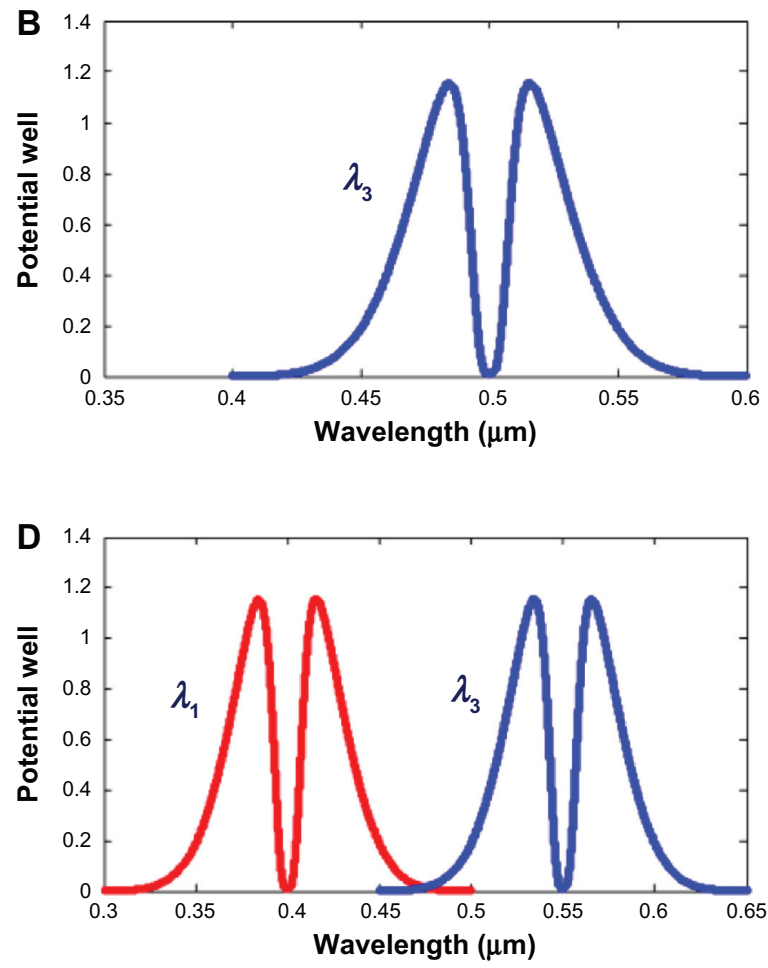

Figure 4 Result of the dynamic tweezers with different wavelengths $(\mathbf{A}-\mathbf{D})$, showing the tunable tweezer by coupling constants, where $R_{a d}=20 \mu \mathrm{m}, R_{R}=R_{L}=5 \mu \mathrm{m}$. 

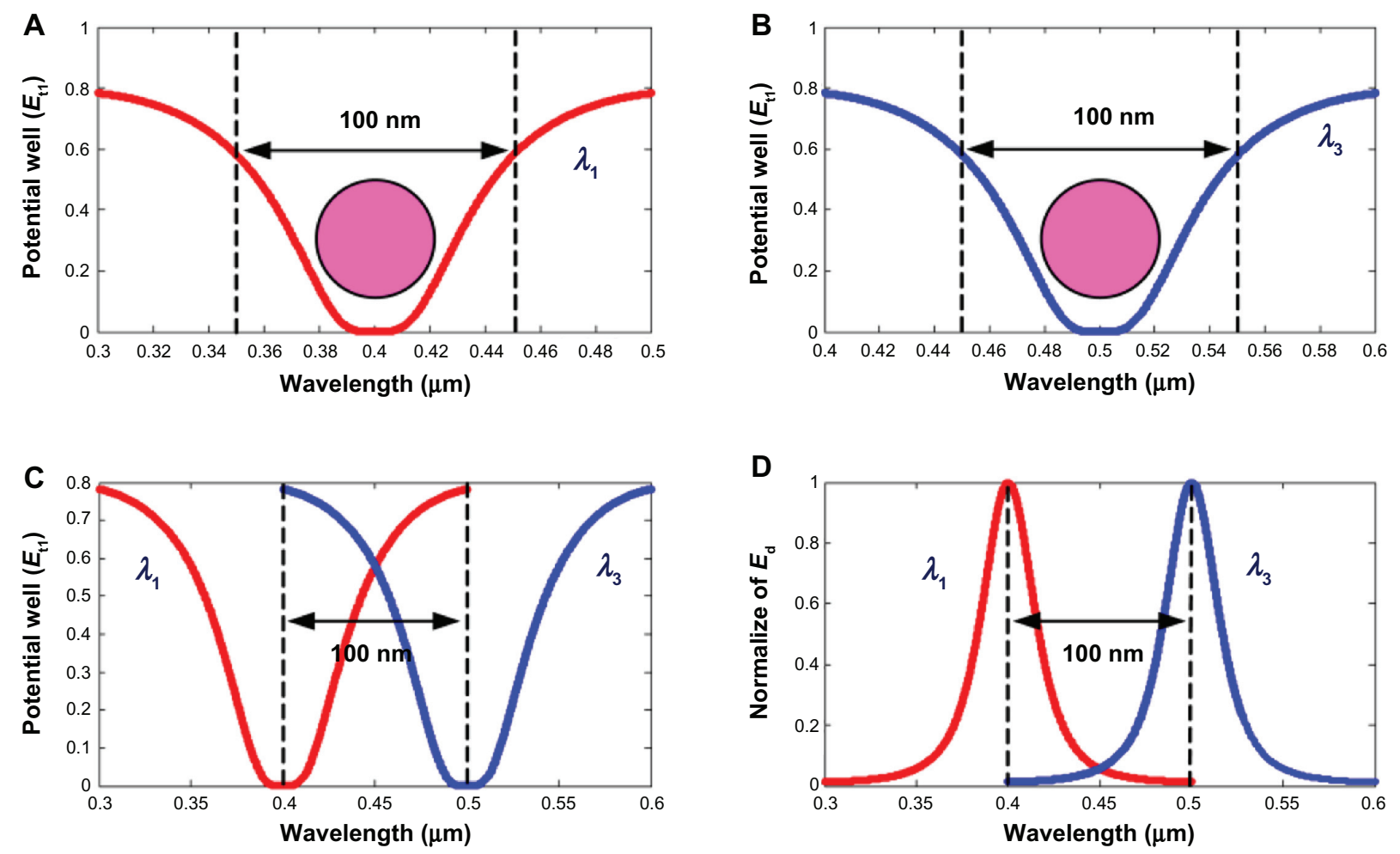

Figure $\mathbf{5}$ Results of the trapping tools, where $\mathbf{A}$ and $\mathbf{B}$ show different sizes and wavelengths, $\mathbf{C}$ shows tweezer separation, and $\mathbf{D}$ shows normalized tweezers, where $R_{a d}=20 \mu \mathrm{m}, R_{R}=R_{L}=5 \mu \mathrm{m}$. The coupling coefficients are $\kappa_{0}=0.5, \kappa_{1}=0.35, \kappa_{2}=0.1$, and $\kappa_{3}=0.35$. The input power is IW, $R_{a d}=20 \mu \mathrm{m}, R_{L}=R_{R}=5 \mu \mathrm{m}$, (A) wavelength $=400 \mathrm{~nm}$, (B) wavelength $=500 \mathrm{~nm},($ C) wavelengths $=400,500 \mathrm{~nm}$ at through port, (D) wavelengths $=400,500 \mathrm{~nm}$ at drop port.

PANDA microring (as shown in Figure 5B). In addition, the tap coefficient, which is used in the crosstalk calculation, follows that of Ramaswami and Liu. ${ }^{11}$

\section{Molecular network performance}

According to the network parameters presented, ${ }^{28-30}$ the proposed network topologies are compared, as shown in Table 1. Three parameters are used to calculate the crosstalk of each node in decibel units $\left(F C_{j}\left(\lambda_{i}\right)\right)$, in which the crosstalk and SNR are used to evaluate the efficiency of each topology. The simulation parameters are kept at the same values for the comparison, otherwise different results would have

Table I The comparison of crosstalk and SNR value of three network topologies

\begin{tabular}{llll}
\hline Parameters & Methods & \\
\cline { 2 - 4 } & $\begin{array}{l}\text { Bus } \\
\text { network }\end{array}$ & $\begin{array}{l}\text { Ring } \\
\text { network }\end{array}$ & $\begin{array}{l}\text { Star } \\
\text { network }\end{array}$ \\
\hline Tap coefficient (dB) & 3 & 3 & 3 \\
Insertion loss (dB) & 15 & 31.3 & 16.9 \\
Number of neural nodes & 10 & 10 & 10 \\
$F C_{j}\left(\lambda_{i}\right)(\mathrm{dB})$ & $3.0 \mathrm{I}$ & 3.01 & 3.01 \\
Crosstalk ratio $(\%)$ & $8.595 \mathrm{e}-2 \mathrm{I}$ & $4.308 \mathrm{e}-37$ & $1.082 \mathrm{e}-22$ \\
SNR $(\mathrm{dB})$ & 5.23 & 5.69 & 4.95 \\
\hline
\end{tabular}

Abbreviations: FC, fiber channel; SNR, signal to noise ratio. been obtained. The topology for a higher SNR and lower crosstalk effect ratio is more reliable for drug delivery and diagnosis network requirements. In the star network, the performance of each node is the same, which reduces the tendency of network failure by connecting the whole system to a central node. Hence, the failure of a transmission line linking any peripheral node to the central node does not affect the rest of the system. In the star topology, the data package or message is transmitted quickly and does not pass through any unnecessary node. The star network requires less cable than a mesh network, but each node must be linked to the central hub.

For the ring network, the signal passes through each network node containing the amplifier, so that the signal is amplified and increased in quality factor $(Q)$; that is, the signal keeps improving as it passes through the successive nodes. However, in a ring network, the failure of a single node can disable the entire network and unidirectional traffic becomes a disadvantage of this topology. To overcome this problem, a fiber distributed data interconnection network is applied for the data package, so that, in case of a ring break, the data package or message is wrapped back onto the complementary ring before reaching the end of the cable ring, as indicated by the name "token ring network." In bus network 
topology, the quality of signal decreases with a decrease in input signal power, and the power penalty goes on increasing; in other words, performance goes down as additional nodes are added to the network. The decrease in quality factor is due to nonuniform power distribution among nodes in the network. However, the main limitation of this topology is cable length and the number of nodes. If a cable is broken, the entire network goes down and the maintenance cost can be very high in the long run.

\section{Conclusion}

This paper proposes the use of network topology for drug delivery and diagnosis network design. The quality of network reliability is calculated in terms of crosstalk effect and SNR value. The experimental results show that the performance of the ring and star network is better than the optical bus network because of fewer values of power loss, crosstalk, and SNR factors. In simulation, all device parameters were chosen specifically so that the practical device that could be fabricated and constructed. In practical application, the small network can be designed by using thin film technology. The molecular network device can be fabricated and embedded within the human body and the required molecular diagnosis can be realized, which will be the subject of future investigation.

\section{Acknowledgments}

The authors would like to thank the Institute of Advanced Photonics Science, Nanotechnology Research Alliance, University of Technology Malaysia, Malaysia, and King Mongkut's Institute of Technology, Thailand, for providing the research facilities. This research was supported by the University of Technology Malaysia's Tier 1/Flagship Research Grant, MyBrain15 Fellowship/MOHE SLAB Fellowship, and a Ministry of Higher Education research grant.

\section{Disclosure}

Other than the support received outlined in the Acknowledgments, the authors have no conflicts of interest to declare in this work.

\section{References}

1. Kotsavasiloglou C, Kalampokis A, Argyrakis P, Baloyannis S. Modeling signal transmission and robustness in biological neural networks. Int $J$ Biol Biomed Eng. 2007;1(2):41-45.

2. Moritani Y, Hiyama SXS, Suda T. Molecular communication for health care applications. In: The Institute of Electrical and Electronics Engineers. Proceedings: Fourth Annual IEEE International Conference on Pervasive Computing and Communications Workshops; March 13-17, 2006, Pisa, Italy. Los Alamitos, CA: IEEE Computer Society; 2006:1-5.
3. Bures P, Huang Y, Oral E, Peppas NA. Surface modifications and molecular imprinting of polymers in medical and pharmaceutical applications. J Control Release. 2001;72(1-3):25-33.

4. Mitatha S, Moongfangklang N, Jalil MA, et al. Proposal for Alzheimer's diagnosis using molecular buffer and bus network. Int J Nanomed. 2011; 2011(6):1209-1216.

5. Mitatha S, Moongfangklang N, Jalil MA, Suwanpayak N, Ali J, Yupapin PP. Multi-access drug delivery network and stability. Int $J$ Nanomed. 2011;6:1757-1764.

6. Glass M. ANSI/IEEE 1073: Medical Information Bus (MIB). Health Informatics J. 1998;4(2):72-83.

7. Schleifer A. Medical information bus. Computer and Information Science. 2010:1-12.

8. Franklin DF, Ostler DV. The P1073 medical information bus. IEEE Micro. 1989;9(5):52-60.

9. ISO/IEEE11073-20101:2004(E). Health informatics - Point-of-care medical device communication - Part 20101: Application profile - base standard.

10. ISO/IEEE11073-20601:2010. Health informatics - Personal health device communication - Part 20601: Application profile - Optimized exchange protocol.

11. Ramaswami R, Liu K. Analysis of effective power budget in optical bus and star networks using erbium-doped fiber amplifiers. J Lightwave Technol. 1993;11(11):1863-1871.

12. Singh S. Performance comparison of optical network topologies in the presence of optimized semiconductor optical amplifiers. JOpt Commun Netw. 2009;1(4):313-323.

13. Randhawa R, Sohol JS. Comparison of optical network topologies for wavelength division multiplexed transport networks. Int J Light Electron Opt. 2010;121(12):1096-1110.

14. Pountourakis IE. Performance evaluation with receiver collisions analysis in very high-speed optical fiber local area networks using passive star topology. J Lightwave Technol. 1998;16(2): 2303-2310.

15. Barranco M, Proenza J, Almeida L. Quantitative comparison of the error-containment capabilities of a bus and a star topology in CAN networks. IEEE Trans Indus Electron. 2011;58(3):802-813.

16. Jalil MA, Tasakorn M, Suwanpayak N, Ali J, Yupapin PP. Nanoscopic volume trapping and transportation using a PANDA ring resonator for drug delivery. IEEE Trans Nanobioscience. 2011;10(2): $106-112$.

17. Neuman KC, Block SM. Optical trapping. Rev Sci Instrum. 2004;75(9): 2787-2809.

18. Svoboda K, Block SM. Biological applications of optical forces. Annu Rev Biophys Biomol Struct. 1994;23(1):247-282.

19. Rosenberry MA, Reyes JP, Tupa D, Gay TJ. Radiation trapping in rubidium optical pumping at low buffer-gas pressures. Phys Rev A. 2007;75:2. Doi: 10.1103/PhysRevA.75.023401.

20. Chen SY, Hu SH, Liu DM, Kuo KT, inventors. Drug delivery nanodevice, its preparation method and used thereof. United States patent application US 20110014296 A1. July 17, 2009.

21. Wagner SS. Optical amplifier applications in fiber optic local network. IEEE Trans Commun. 1987;35(4):419-426.

22. Enomoto A, Moore MJ, Suda T, Oiwa K. Design of self-organizing microtubule networks for molecular communication. Nanocommun Net. 2011;2(1):16-24.

23. Smit MK. Progress in AWG design and technology. In: Proceedings of WFOPC2005: 4th IEEE/LEOS Workshop on Fibres and Optical Passive Components. Mondello (Palermo), Italy, June 22-24. 2005:26-31.

24. Chen YK. Amplified distributed reflective optical couplers. IEEE Photon Tech Lett. 1992;4(6):570-573.

25. Ross FE. An overview of FDDI: the fiber distributed data interface. IEEE J Sel Areas Commun. 1989;7(7):1043-1051.

26. Willner AE, Hwang SM. Transmission of many WDM channels through a cascade of EDFA's in long distance links and ring networks. J Lightwave Technol. 1995;13(5):802-816. 
27. Piyatamrong B, Kulsirirat K, Mitatha S, Yupapin PP. Dynamic potential well generation and control using double resonators incorporating in an add/drop filter. Mod Phys Lett B. 2010;24(32):3071-3082.

28. Ota T. Satellite coupler: application to distributed star network. Electron Lett. 1994;3(24):2060-2062.

29. Lafata P, Vodrazka J. Application of passive optical network with optimized bus topology for local backbone data network. Microwave and Optical Technology Letters. 2011;53(10):2351-2355.
30. Chang $\mathrm{CH}$, Liang TC, Huang CY. DWDM self-healing access ring network with cost-saving, crosstalk-free and bidirectional OADM in single fiber. Optic Commun. 2009;282(23):4518-4523.

\section{Publish your work in this journal}

The International Journal of Nanomedicine is an international, peerreviewed journal focusing on the application of nanotechnology in diagnostics, therapeutics, and drug delivery systems throughout the biomedical field. This journal is indexed on PubMed Central, MedLine, CAS, SciSearch ${ }^{\circledR}$, Current Contents ${ }^{\circledR} /$ Clinical Medicine,
Journal Citation Reports/Science Edition, EMBase, Scopus and the Elsevier Bibliographic databases. The manuscript management system is completely online and includes a very quick and fair peer-review system, which is all easy to use. Visit http://www.dovepress.com/ testimonials.php to read real quotes from published authors. 